\title{
The Effects of Acute Exercise on Subsequent Cigarette Smoking
}

\author{
Ovide F. Pomerleau, ${ }^{1,6}$ Herbert H. Scherzer, ${ }^{2}$ \\ Neil E. Grunberg, ${ }^{3}$ Cynthia S. Pomerleau, ${ }^{1}$ \\ James Judge, ${ }^{4}$ Joanne B. Fertig, ${ }^{5}$ and Joseph Burleson ${ }^{5}$ \\ Accepted for publication: December 10, 1985
}

The present study was conducted to examine the effects of acute aerobic exercise on smoking behavior. On alternate days, 10 healthy young smokers were subjected to half an hour of sustained high exercise (about $56 \%$ of maximum work capacity) or of low exercise (about $28 \%$ of maximum, simulating normal daytime activity). During the high-exercise condition, there were pronounced increases in physiological markers of physical activity such as mean work, heart rate, and lactic acid as well as elevations in circulating hormones (norepinephrine, epinephrine, and immunoreactive beta-endorphin and cortisol) known to be affected by vigorous exercise. Despite a trend toward decreased desire for cigarettes after the high exercise condition, there were

The authors acknowledge with gratitude the assistance of Judith L. Cooney, Ned Cooney, Nancy Haley, Mark Majchrzak, Stephanie M. Nespor, and Edward Shaskan. Partial support for this research was provided by National Cancer Institute Grant CA/DA 42730, by the Arthur Thornton Fund, and by the New Britain General Hospital Auxiliary.

${ }^{1}$ Behavioral Medicine Program, Department of Psychiatry, University of Michigan School of Medicine, Ann Arbor, Michigan.

${ }^{2}$ Human Performance Laboratory, New Britain General Hospital, and Department of Medicine, University of Connecticut School of Medicine, New Britain, Connecticut.

${ }^{3}$ Department of Medical Psychology, Uniformed Services University of the Health Sciences, Bethesda, Maryland.

${ }^{4}$ Department of Medicine, University of Connecticut School of Medicine, Farmington, Connecticut. ${ }^{5}$ Department of Behavioral Biology, Walter Reed Army Institute of Research, Washington, D.C. ${ }^{6}$ To whom correspondence should be addressed at Behavioral Medicine Program, Department of Psychiatry, University of Michigan School of Medicine, Riverview Building, 900 Wall Street, Ann Arbor, Michigan 48105. 
no differences in plasma nicotine levels following the smoking of a usualbrand cigarette $35 \mathrm{~min}$ later. The sustained effects of the two exercise conditions were also similar: plasma cotinine levels $24 \mathrm{hr}$ later (reflecting nicotine intake over the entire exercise day) revealed no significant differences between high and low exercise.

KEY WORDS: cigarette smoking; cotinine; exercise; nicotine; stress hormones.

\section{INTRODUCTION}

Numerous pathological conditions are associated with cigarette smoking, the most devastating of which involve the cardiovascular and pulmonary systems (Clee and Clark, 1984). Despite persistent and imaginative efforts to identify and manipulate various social, psychological, and biological factors, no more than one-fourth to one-third of treated smokers achieve longterm abstinence (Evans and Lane, 1980; Lichtenstein and Brown, 1982; Pechacek, 1977). There is therefore a need to identify procedures and activities that might serve as behavioral alternatives or substitutes for smoking and that can either interfere with or provide some of the reinforcement derived from nicotine (for example, by mimicking some of the biochemical and physiological effects of smoking).

Several epidemiological studies have noted a correlation between physical exercise and reduced cigarette smoking (Hickey et al., 1975; Cheraskin and Ringsdorf, 1971). A similar relationship was seen in an assessment of a running club whose members averaged 35 miles per week (Morgan et al., 1976). The investigators found that of 141 members, 35 had been smokers when they joined the club, and all but 3 quit smoking. While a causal relationship cannot be inferred, these reports and various uncontrolled observations of the apparent incompatibility of exercise and smoking suggest that the therapeutic potential of aerobic exercise should be evaluated more systematically.

We conducted the present study as a preliminary attempt to examine the effects of exercise on smoking, monitoring both physiological and biochemical changes. On alternate days, after half an hour of either intense exercise or low exercise intended to simulate normal daytime activity, subjects were asked to smoke a cigarette of their usual brand. Validation of the intensity of physical activity was provided by standard physiological markers such as mean work output, heart rate, and lactic acid as well as by the measurement of hormones (norepinephrine, epinephrine, immunoreactive betaendorphin, and cortisol) known to be affected by exercise (Carr and Fishman, 1985; Hartley et al., 1972). Half an hour after the termination of exercise, subjects were asked to smoke a cigarette in order to determine the effects 
of exercise on nicotine intake as measured by plasma nicotine. A longer-term assessment of the impact of exercise on smoking was based on measures of plasma cotinine [a nicotine metabolite with a half-life of $30 \mathrm{hr}$ (Matsukura et al.,1979)], taken $24 \mathrm{hr}$ after each session to provide an integrated measure of nicotine intake for the day. The Shiffman Withdrawal Scale (Shiffman and Jarvik, 1976) was administered to determine desire to smoke (craving) and physiological and psychological withdrawal symptoms (Pomerleau et al., 1983b) before and after exercise. The Profile of Mood States (McNair et al., 1971) was also administered at these times to assess mood and other subjective states.

\section{METHOD}

\section{Subjects}

Ten male smokers, in good health and neither taking medications nor participating regularly in organized athletic activity, were recruited from the local community. They were paid $\$ 25$ per session for two 2 -hr sessions in 1 week. Data obtained during the screening interview and during exercise testing on a bicycle ergometer were used to determine fitness level. Subjects had a mean age of $24.2 \pm 1.6$ years and had smoked for $8.3 \pm .9$ years. Average height was $177.5 \pm 1.3 \mathrm{~cm}$ and weight was $75.4 \pm 13.9 \mathrm{~kg}$. Subjects reported smoking $28.5 \pm 1.9$ cigarettes per day; plasma cotinine values, $357.0 \pm 36.9 \mathrm{ng} / \mathrm{ml}$, characterize them as heavy smokers (Pomerleau et al., 1983b). Maximum work capability, $4.2 \pm .2 \mathrm{~W} / \mathrm{kg}$, and maximum aerobic capability $\left(V \mathrm{O}_{2} \max \right), 40.6 \pm 2.7 \mathrm{ml} / \mathrm{kg} / \mathrm{min}$, suggest that the subjects as a group were not aerobically fit compared with others their age (Folinsbee et al., 1983; Harley et al., 1972; Mellerowik et al., 1981). None of the subjects evinced an interest in quitting smoking.

\section{Experimental Apparatus}

Subjects were tested in a human performance laboratory using a computerized system for cardiopulmonary evaluation (Medical Graphics System 2000 and Cambridge polygraph). The bicycle ergometer (Meinhardt KEM-2) generated an electronically braked, continually variable workload and provided direct data input to a dedicated computer that calculated physical work, ventilation, oxygen consumption, and carbon dioxide levels on-line. With nostrils occluded, the subjects breathed though a mouthpiece connected to a Fleich pneumotach, allowing direct calculation of respiratory parameters. 
Lactic acid was analyzed by enzymatic oxidation/collimetry using a Dupont ACA Analyzer.

\section{Plasma Collection and Analysis}

Blood samples for analysis of nicotine and norepinephrine, epinephrine, beta-endorphin, and cortisol were drawn from a superficial anterior vein of the subject's arm using an 18-gauge catheter. Ten-milliliter samples of whole blood were taken every $10 \mathrm{~min}$ (starting $20 \mathrm{~min}$ into the session and ending with the 130-min sample, except during the actual period of exercise). Blood was collected in plastic tubes containing EDTA and immediately stored in ice water. Samples were centrifuged at $4^{\circ} \mathrm{C}$ within $1 \mathrm{~min}$, and plasma aliquots were immediately stored at $-80^{\circ} \mathrm{C}$.

Plasma nicotine and cotinine were measured by radioimmunoassay (RIA). The interassay and intraassay coefficients of variation were $6 \%$, and the lower limit of detectability was $.37 \mathrm{ng} / \mathrm{ml}$ (Haley et al., 1983; Hill et al., 1983). Beta-endorphin was measured using an RIA kit supplied by ImmunoNuclear (Stillwater, Minn.). The sensitivity of the assay was $10 \mathrm{pg} / \mathrm{ml}$, the intraassay coefficient of variation was $10 \%$, and the extraction efficiency of added beta-endorphin from plasma was $95-110 \%$. These assays were conducted at the American Health Foundation (Valhalla, N.Y.) and have been described in previous reports (Pomerleau et al., 1983a; Seyler et al., 1984). Catecholamines (norepinephrine and epinephrine) were measured by radioenzymatic assay (Durrett and Ziegler, 1980). The sensitivity was $2 \mathrm{pg} / \mathrm{ml}$ for norepinephrine and $3 \mathrm{pg} / \mathrm{ml}$ for epinephrine. Cortisol was assayed with an RIA kit purchased from Clinical Assays, Cambridge, Mass.; the assay sensitivity was $2.4 \mathrm{ng} / \mathrm{ml}$. These assays were conducted at the Uniformed Services University of the Health Sciences (Bethesda, Md.).

\section{Procedure}

From an initial 5-ml blood sample taken in a screening session a few days before participation, the hematocrit was determined ( $\geq 42 \%$ was required for participation) and plasma cotinine was used to provide an objective determination of usual nicotine intake and smoking status. Subjects were told that the object of the study was to examine the effects of exercise on circulating lactic acid levels in smokers. They were told to consume similar breakfasts (including number of cups of coffee) before each session. Experimental sessions were conducted in the late morning. On an experimental day, subjects smoked ad libitum until just before the start of a session. Each session lasted $130 \mathrm{~min}$. After a 30-min rest period, there was $30 \mathrm{~min}$ of exer- 
cise. Ninety-five minutes after the start of the session (35 min after exercising), there was was a 5-min smoking period. Questionnaires on subjective state were filled out 10 and 80 min after the start of the session. Sessions were conducted on alternate days; the order was counterbalanced across subjects, so that five subjects performed the low-exercise procedure on the first day, while the other five performed the high-exercise procedure on the first day.

The high-exercise condition involved $10 \mathrm{~min}$ of threshold determination, using an increasing exercise load on the bicycle ergometer to establish maximum aerobic capability $\left(\mathrm{VO}_{2} \max\right.$ ) (Davis et al., 1982); the remaining 20 min was spent attempting to maintain exercise level up to $80 \%$ of that maximum $\left(80 \% V \mathrm{O}_{2}\right.$ max). The control condition involved adjusting the bicycle ergometer brake load to keep the cardiac rate at about $100 \mathrm{bpm}$ (about $30 \%$ $V \mathrm{O}_{2}$ max). When subjects were not exercising, they sat in an easy chair, where they read magazines and filled out questionnaires. Twenty-four hours after each of the two exercise sessions, subjects reported to the screening laboratory, where a $5-\mathrm{ml}$ blood sample was taken for cotinine analysis. Three months after the experiment, changes in smoking habit and exercise were queried by telephone.

\section{RESULTS}

Statistical comparisons were based on repeated-measures ANOVA (Dixon, 1981), with exercise level as a within-subjects factor and, in cases where multiple samples were obtained, time as an additional within-subjects factor. All analyses included an initial evaluation for order effects, using counterbalancing sequence as a between-groups variable. No significant order effects were found for nicotine intake or for physiological, hormonal, and subjective measures.

The effects of half an hour of vigorous aerobic exercise were pronounced, as shown in Table I. All statistical comparisons of the two conditions were highly significant: mean work $[F(1,8)=154.7, p<.001]$, percentage of maximum work $[F(1,8)=275.3, p<.001]$, heart rate in the final minute $[F(1,8)=275.3, p<.001]$, heart rate in the final minute $[F(1,8)=247.6$, $p<.001]$, and lactic acid $[F(1,8)=104.5, p<.001]$. The effects of vigorous exercise on norepinephrine, epinephrine, and immunoreactive beta-endorphin and cortisol are shown in Fig. 1. With the exception of cortisol, the levels of these hormones were significantly elevated following high exercise (time course based on the five samples taken between 20 and $80 \mathrm{~min}$ ): norepinephrine $[F(4,32)=26.8, p<.001]$, epinephrine $[F(4,32)=6.9, p$ 

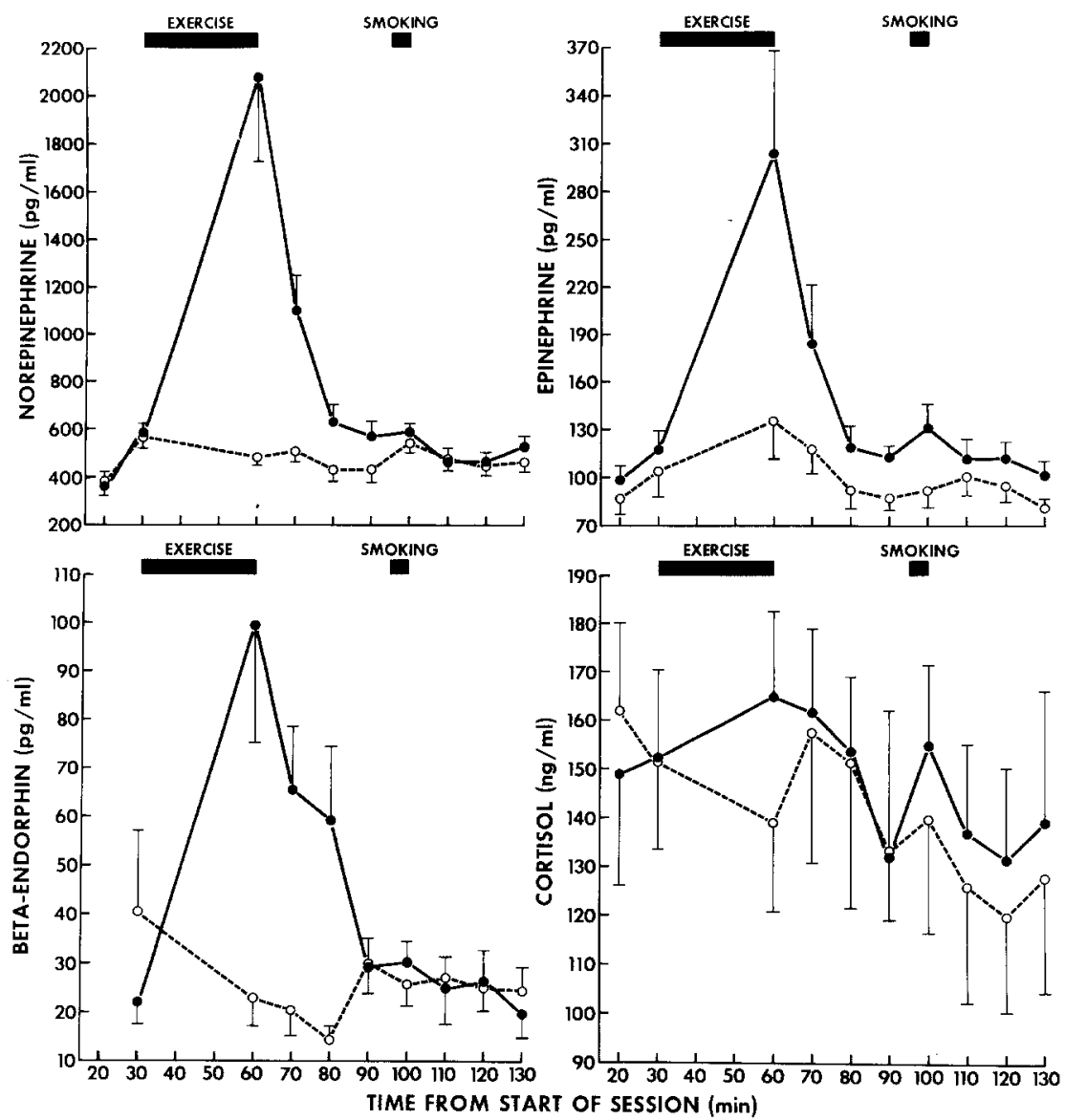

Fig. 1. Effects of exercise and smoking on circulating hormones; high exercise is indicated by solid lines and low exercise by dashed lines. Means $\pm \mathrm{SE} ; N=10$.

$<.001]$, and beta-endorphin $[F(4,32)=7.6, p<.001]$. The highest levels were observed immediately after high exercise. Although causal inference is limited by the fact that smoking was not controlled experimentally, smoking a cigarette was associated with small but significant elevations in plasma norepinephrine and epinephrine (time-course analysis based on the five samples taken between 90 and $130 \mathrm{~min}$, using the 90 -min sample as a covariate to control for elevated values following high exercise): norepinephrine $[F(3,24)=5.7, p<.01]$ and epinephrine $[F(3,24)=6.0, p<.01]$. There was also a significant increase in feelings of tiredness [Profile of Mood States Energetic/Tired Scale, $F(1,7)=11.5, p<.01]$. 
Table I. Physiological Effects of High and Low Exercise (Mean \pm

\begin{tabular}{|c|c|c|}
\hline \multicolumn{3}{|c|}{ Exercise level } \\
\hline & High & Low \\
\hline $\begin{array}{l}\text { Mean work over exercise session } \\
\text { (W/kg body weight) }\end{array}$ & $2.4 \pm$ & $1.2 \pm .1$ \\
\hline $\begin{array}{l}\text { Percentage of maximum work } \\
(\text { mean work } / \text { maximum work } \times 100)\end{array}$ & $56.1 \pm 1.6$ & $27.5 \pm .9$ \\
\hline $\begin{array}{l}\text { Heart rate in final minute of exercise } \\
\text { session (bpm) }\end{array}$ & $178 \pm$ & 101 \\
\hline $\begin{array}{l}\text { Lactic acid after exercise session } \\
(\mathrm{Meq} / \mathrm{L})\end{array}$ & $9.2 \pm$ & $.7 \pm .2$ \\
\hline
\end{tabular}

Level of exercise had little effect on subsequent nicotine intake, as shown in Fig. 2. Despite a trend toward decreased desire for cigarettes following high exercise [Shiffman Craving Subscale, $F(1,8)=3.72, p<.09$ ], the time course for plasma nicotine for the two exercise conditions was not significantly different; cotinine levels also did not differ significantly. In both the highand the low-exercise conditions, the highest levels of plasma nicotine were observed at the termination of cigarette smoking; compared to presmoking levels, these elevations over time were highly significant $[F(4,32)=13.7, p$ $<.001]$. Finally, no changes in either exercise or smoking habits were reported by the subjects at a 3 -month follow-up.

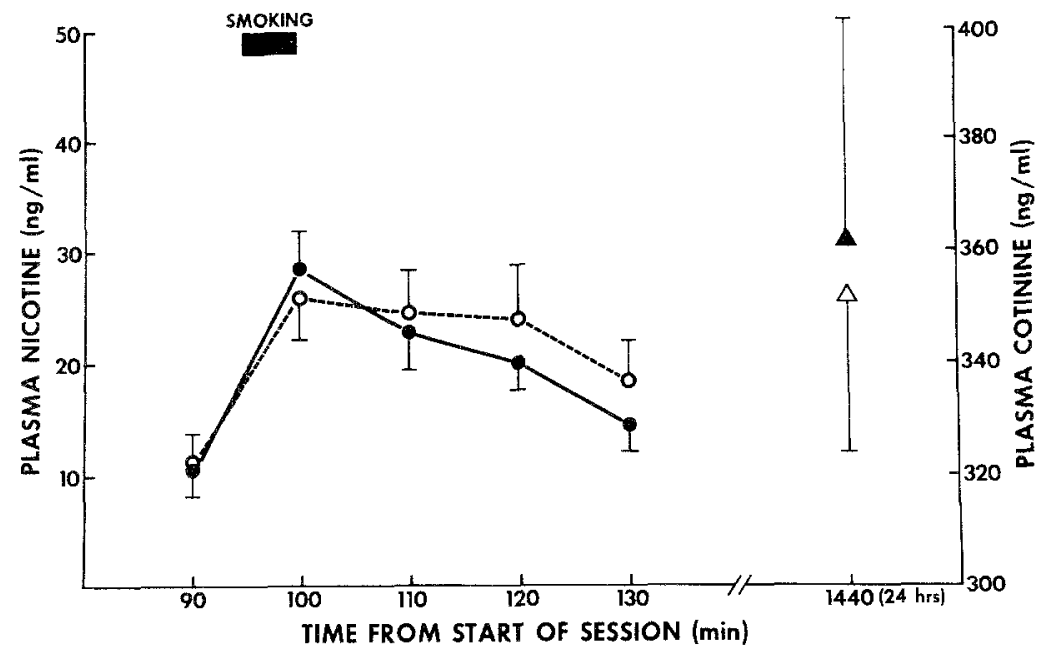

Fig. 2. Plasma nicotine and cotinine following exercise; nicotine is indicated by circles, cotinine by triangles, high exercise by solid lines, and low exercise by dashed lines. Means $\pm \mathrm{SE} ; N=10$. 


\section{DISCUSSION}

In the final $20 \mathrm{~min}$ of the high-exercise condition, the subjects sustained a work level that was $56 \%$ of their maximum aerobic capacity. Taking into account lactate production and the physiological markers of physical activity as well as subjective reports of exhaustion, this output probably represents the maximum sustainable workload for these relatively unfit subjects (Karlson, 1971).

The pronounced elevations in hormones known to be affected by vigorous exercise provide further corroboration of the intensity of the exertion (Carr and Fishman, 1985). These same hormones have been classified as constituting part of the stress response (Selye, 1980). The magnitude and time course for norepinephrine and epinephrine in the present study are in keeping with values promulgated for the catecholamine response to $8 \mathrm{~min}$ of intense aerobic exercise in untrained subjects (Hartley et al., 1972). The sharp increase in plasma beta-endorphin immunoreactivity following the highexercise condition clearly confirms, using subjects as their own controls with well-defined work parameters, previous reports indicating increased levels of this neuropeptide in circulation following sustained aerobic activity (Carr et al., 1981; Farrell et al., 1982). In the present study, cortisol immunoreactivity, although somewhat elevated following high exercise, was too variable to produce statistically significant trends. The overall levels, however, are consistent with those observed following half an hour of graded exercise in unfit subjects (Bloom et al., 1976).

Despite these well-defined physiological and hormonal changes, however, the level of exercise had a negligible impact on smoking half an hour later. Even though there was a trend toward decreased desire for cigarettes, and even though 3 of the 10 subjects did ask if they were required to smoke following the high-exercise condition, there were no significant differences in plasma nicotine after smoking a usual-brand cigarette. Possibly the request that subjects light up a cigarette overwhelmed an initial disinclination to smoke following intense exercise-that is, the presentation of a cigarette may have provided salient cues for smoking, enhanced by an hour and a half of abstinence. Thus, the present design did not permit a test of the effect of exercise on the decision to smoke. The sustained effects of the two exercise conditions on nicotine intake, however, were similar: cotinine values $24 \mathrm{hr}$ later (reflecting nicotine intake over the entire exercise day) revealed no significant differences between high- and low-exercise sessions.

It may be that subjects would have smoked less over the course of the day had they not in fact "resumed" smoking in compliance with experimental procedure. On the other hand, the initial disinclination to smoke may simply have been a response to the extreme discomfort produced by high 
exercise in these untrained subjects, in which case a normal smoking pattern would be reinstated once the discomfort had abated. In view of its potential clinical usefulness, however, the finding of a trend toward reduced desire to smoke following high exercise deserves further investigation in the context of an experiment that would permit evaluation of the relationship between desire sustained over a longer period of time and decision to smoke.

At face value, these results, based on a relatively small number of subjects, do not confirm previous reports (e.g., Howley et al., 1980; Morgan et al., 1976) suggesting that exercise is incompatible with smoking. Several critical points, however, remain unresolved. In the present study, the exercise was acute and intense and, in physically inactive subjects, may have functioned more as a severe stressor than as either a substitute for or an inhibitor of smoking. A more definitive test may be provided by studying the effects of chronic exercise, particularly in subjects expressing an interest in reducing or quitting smoking. Physiological and psychological differences in the response to physical exercise are quite pronounced between aerobically fit and aerobically unfit subjects. For example, during exercise, regular exercisers exhibit greater plasma glucose but lower plasma catecholamine and lactic acid levels than do unfit subjects (Bloom et al., 1976; Hartley et al., 1972). People who are physically fit also exhibit more rapid heart-rate recovery and lower levels of anxiety following psychological stress (Sinyor et al., 1983). Thus, the sense of psychological well-being attributed to exercise may be a manifestation of physical conditioning, reflecting adaptation to exertion (Folkins and Sime, 1981; Hughes, 1984). It may be that the biochemical and physiological response to chronic exercise more closely mimics the response to chronic smoking than does the response to acute exercise (Pomerleau and Rodin, 1986). Cognitive and motivational factors may also play a role, in that people who come to enjoy the benefit of chronic exercise may perceive that smoking undercuts their ability to exercise.

But even if regular exercise does have the potential to serve as a healthful alternative to smoking, smokers may have to be taught to use the changes elicited by exercise to compensate for those produced by smoking. The behavior of lighting up a cigarette in response to stress and to the various demands of daily living is highly entrained (Ashon and Stepney, 1982; Mangan and Golding, 1985; Pomerleau and Pomerleau, 1984), and its strength should not be underestimated.

\section{REFERENCES}

Ashton, H., and Stepney, R. (1982). Smoking: Psychology and Pharmacology, Tavistock, London. 
Bloom, S. R., Johnson, R. H., Park, D. M., Rennie, M. J., and Sulaiman, W. R. (1976). Differences in the metabolic and hormonal response to exercise between racing cyclists and untrained individuals. J. Physiol. 258: 1-18.

Carr, D. B., and Fishman, S. M. (1985). Exercise and the endogenous opioids. In Fotherby, K., and Pal, S. (eds.), Exercise Endocrinology, De Gruyter, New York.

Carr, D. B., Bullen, B. A., Skrinar, G. S., Arnold, M. a., Rosenblatt, M., Beitins, I. Z., Martin, J. B., and McArthur, J. W. (1981). Physical conditioning facilitates the exerciseinduced secretion of beta-endorphin and beta-lipotropin in women. N. Engl. J. Med. 305: 560-563.

Cheraskin, E., and Ringsdorf, W. M. (1971). Predictive medicine: Physical activity. J. Am. Geriat. Soc. 19: 969-973.

Clee, M. D., and Clark, R. A. (1984). Tobacco smoking: The medical sequelae. In Balfour, D. J. K. (ed.), Nicotine and the Tobacco Smoking Habit, Pergamon Press, Oxford, pp. 177-198.

Davis, J. A., Whipp, B. J., La Marra, N., Huntsman, D. J., Frank, H., and Wasserman, K. (1982). Effects of ramp slope on determination of aerobic parameters from the ramp exercise test. Med. Sci. Sports Exer. 14: 339-343.

Dixon, W. J. (ed.) (1981). BMDP Statistical Software, University of California Press, Berkeley.

Durrett, L. R., and Ziegler, M. G. (1980). A sensitive reactive assay for catecholamine drugs. J. Neurosci. Res. 5: 587-598.

Evans, D., and Lane, D. S. (1980). Long-term outcome of smoking cessation workshops. Am. J. Publ. Health 70: 725-727.

Farrell, P. A., Gates, W. K., Maksud, M. G., and Morgan, W. P. (1982). Increases in plasma beta-endorphin/beta-lipotropin immunoreactivity after treadmill running in humans. $J$. Appl. Physiol. 52: 1245-1249.

Folinsbee, L. G., Wallace, E. S., Beldi, J. F., and Horvath, S. M. (1983). Exercise respiratory pattern in elite cyclists and sedentary subjects. Med. Sci. Sports Exerc. 15: 503-509.

Folkins, C. H., and Sime, W. E., (1981). Physical fitness training and mental health. Am. Psychol. 36: $373-389$.

Haley, N. J., Axelrad, C. M., and Tilton, K. A. (1983). Validation of self-reported smoking behavior: Biochemical analyses of cotinine and thiocyanate. Am. J. Publ. Health 73: 1204-1207.

Hartley, L. H., Mason, J. W., Hogan, R. P., Jones, L. G., Kotchen, T. A., Mousey, E. H., Wherry, F. E., Pennington, L. L., and Ricketts, P. T. (1972). Multiple hormonal responses to graded exercise in relation to physical training. J. Appl. Physiol. 33: 602-606.

Hickey, N., Mulcahy, R., Bourke, T., Graham, I., and Wilson-Davis, K. (1975). Study of coronary risk factors related to physical activity in 15,171 men. Br. Med. J. 3: 507-509.

Hill, P., Haley, N., and Wynder, E. (1983). Cigarette smoking as a risk for cardiovascular disease. 1. Biobehavioral analyses, carboxyhemoglobin, plasma nicotine, cotinine, and thiocyanate versus self-report smoking data. J. Chron. Dis. 36: 439-449.

Howley, T. J., Callahan, E. J., and Yaeter, R. (1980). Aerobic exercise and the treatment of cigarette smoking. Unpublished manuscript presented at the fourteenth Annual Convention of the Association for the Advancement of Behavior Therapy.

Hughes, J. R. (1984). Psychological effects of habitual aerobic exercise: A critical review. Prevent. Med. 13: 66-78.

Karlson, J. (1971). Lactate and phosphogen concentrations in working muscles of man with special reference to oxygen deficit at the onset of work. Acta Physicol. Scand. Suppl. 358: (entire issue).

Lichtenstein, E., and Brown, R. A. (1982). Current trends in the modification of cigarette dependence. In Bellack, M., Herson, M., and Kazdin, A. E., (eds.), International Handbook of Behavior Modification and Therapy, Plenum Press, New York, pp. 574-604.

Mangan, G. L., and Golding, J. F. (1984). The Psychopharmacology of Smoking, Cambridge University Press, Cambridge.

Matsukura, S., Sakamoto, N. Seino, Y., Tamada, T., Matsuyama, H., and Muramaka, H. (1979). Cotinine excretion and daily cigarette smoking in habituated smokers. Clin. Pharmacol. Ther. 25: 555-561. 
McNair, D. M., Loor, M., and Droppleman, L. F. (1971). Profile of Mood States, Educational and Industrial Testing Service, San Diego, Calif.

Mellerowic, H., and Smodlaka, V. N. (1981). Measurement and evaluation of maximal ergometric power. In Mellerowic, H., and Smodlaka, V. N. (eds.), Ergometry, Basics of Medical Exercise Test, Urban \& Schwarzenberg, pp. 55-61.

Morgan, R., Gildiner, M., and Wright, G. (1976). Smoking reduction in adults who take up exercise: A survey of a running club for adults. J. Can. Assoc. Health Phys. Educ. Recreat. 52: $39-43$.

Pechacek, T. (1979). Modification of smoking behavior. In Krasnegor, N. (ed.), The Behavioral Aspects of Smoking (NIDA Monograph 26), National Institute on Drug Abuse, Rockville, Md., pp. 127-188.

Pomerleau, O.F., and Pomerleau, C.S. (1984). Neuroregulators and the reinforcement of smoking: Towards a biobehavioral explanation. Neurosci. Biobehav. Rev. 8: 502-513.

Pomerleau, O. F., Fertig, J. B., Seyler, L. E., and Jaffe, J. (1983a). Neuroendocrine reactivity to nicotine in smokers. Psychopharmacology 83: 61-67.

Pomerleau, O. F., Fertig, J. B., and Shanahan, S. O. (1983b). Nicotine dependence in cigarette smoking: An empirically based, multivariate model. Pharmacol. Biochem. Behav. 19: $291-299$.

Pomerleau, O. F., and Rodin, J. (1986). Behavioral medicine and health psychology. In Garfield, S. L., and Bergin, A. E. (Eds.), Handbook of Psychotherapy and Behavior Change (3rd ed.). Wiley, New York, pp. 483-522.

Selye, H. (ed.). (1980). Selye's Guide to Stress Research, Vol. I, Van Nostrand Reinhold, New York.

Seyler, L. E., Fertig, J. B., Pomerleau, O. F., Hunt, D., and Parker, K. (1984). The effects of smoking on ACTH and cortisol secretion. Life Sci. 34: 57-65.

Shiffman, S., and Jarvik, M. (1976). Smoking withdrawal symptoms in two weeks of abstinence. Psychopharmacologia 50: 35-39.

Sinyor, D., Schwartz, S. G., Peronnet, F., Brisson, G., and Seraganian, P., (1983). Aerobic fitness level and reactivity to psychosocial stress: Physiological, biochemical and subjective measures. Psychom. Med. 45: 205-217. 